\title{
On the ECI and CEI of Boron-Nitrogen Fullerenes
}

\author{
Tingzeng Wu, Ying Wu \\ School of Mathematics and Statistics, Qinghai Nationalities University, Xining, China \\ Email:ma thtzwu@163.com
}

How to cite this paper: $\mathrm{Wu}, \mathrm{T} . \mathrm{Z}$. and $\mathrm{Wu}$, Y. (2018) On the ECI and CEI of Boron-Nitrogen Fullerenes. Applied Mathematics, 9, 891-896.

https://doi.org/10.4236/am.2018.98061

Received: July 22, 2018

Accepted: August 12, 2018

Published: August 15, 2018

Copyright $\odot 2018$ by authors and Scientific Research Publishing Inc. This work is licensed under the Creative Commons Attribution International License (CC BY 4.0).

\begin{abstract}
The eccentric connectivity index and connective eccentricity index are important topological indices for chemistry. In this paper, we investigate the eccentric connectivity index and connective eccentricity index of boron-nitrogen fullerenes, respectively. And we give computing formulas of eccentric connectivity index and connective eccentricity index of all boron-nitrogen fullerenes with regular structure.
\end{abstract}

\section{Keywords}

Eccentric Connectivity Index, Connective Eccentricity Index, Boron-Nitrogen Fullerenes

\section{Introduction}

All graphs considered in this paper are simple connected graphs. Let $G$ be a graph with vertex set $V(G)$ and edge set $E(G)$. Let $v \in V(G), d(v)$ denotes the degree of $v$. For vertices $u, v \in V(G)$, the distance $d(u, v)$ is defined as the length of a shortest path between $u$ and $v$ in $G$. The eccentricity $\varepsilon(v)$ of a vertex $v$ is the maximum distance from $v$ to any other vertex.

The chemical information derived through the topological index has been found useful in chemical documentation, isomer discrimination, structure-property correlations, etc. For quite some time there has been rising interest in the field of computational chemistry in topological indices. The interest in topological indices is mainly related to their use in nonempirical quantitative structure-property relationships and quantitative structure-activity relationships. Among various indices, the eccentric connectivity index and connective eccentricity index involving eccentricity have attracted much attention.

Sharma et al. [1] introduced a distance-based molecular structure descriptor, 
the eccentric connectivity index (ECI for short) defined as

$$
\xi^{c}(G)=\sum_{v \in V(G)} d(v) \varepsilon(v) .
$$

The ECI was successfully used for mathematical models of biological activities of diverse nature, see [2]-[7] and the references cited therein.

A novel graph invariant for predicting biological and physical properties-connective eccentricity index (CEI briefly) was introduced by Gupta et al. [8], which was defined as:

$$
\xi^{c e}(G)=\sum_{v \in V(G)} \frac{d(v)}{\varepsilon(v)} .
$$

Some recent results on the CEI of graphs can be found in [9]-[14].

Boron-nitrogen fullerene is a member of the fullerene family, it has been extensively studied [15] [16] [17]. A boron-nitrogen fullerene is also called $(4,6)$-fullerene graph which is a plane cubic graph whose faces have sizes 4 and 6 . Let $G$ be a $(4,6)$-fullerene graph with $n$ vertices. By Euler's formula, $G$ has exactly six faces of size 4 and $\frac{n}{2}-4$ faces of size 6 .

A $(4,6)$-fullerene graph is said to be of dispersive structure if has neither three squares arranged in a line nor a square-cap. According to the quadrilateral positional relationship, Wei and Zhang [18] given a classification of all $(4,6)$-fullerenes as follows.

Lemma 1 [18] Let $G$ be a (4,6)-fullerene graph. Then $G$ can be of one of the following five types:

1) a cube,

2) a hexagonal prism,

3) a tubular graph with at least one hexagon-layer,

4) a (4,6)-fullerene graph of lantern structure,

5) a (4,6)-fullerene graph of dispersive structure, where the resulting graphs see Figure 1.

In this paper, we aim to investigate the ECI and CEI of a boron-nitrogen fullerene. In next section, we give computing formulas of ECI and CEI of a boron-nitrogen fullerene.

\section{Computing Formula of ECI and CEI of a $(4,6)$-Fullerene}

In this section, according the classification of all boron-nitrogen fullerenes in Lemma 1, we will give computing formula of CEI of a $(4,6)$-fullerene.

Theorem 2 Let $G$ be a $(4,6)$-fullerene graph. Then

1) $\xi^{c e}(G)= \begin{cases}8 & \text { if } G \text { is a cube, } \\ 9 & \text { if } G \text { is hexagonal prism. }\end{cases}$

2) $\xi^{c}(G)= \begin{cases}72 & \text { if } G \text { is a cube, } \\ 144 & \text { if } G \text { is hexagonal prism. }\end{cases}$ 


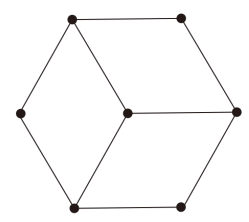

a cube

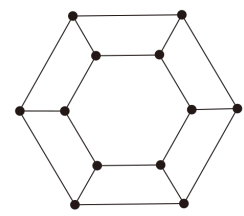

a hexagonal prism

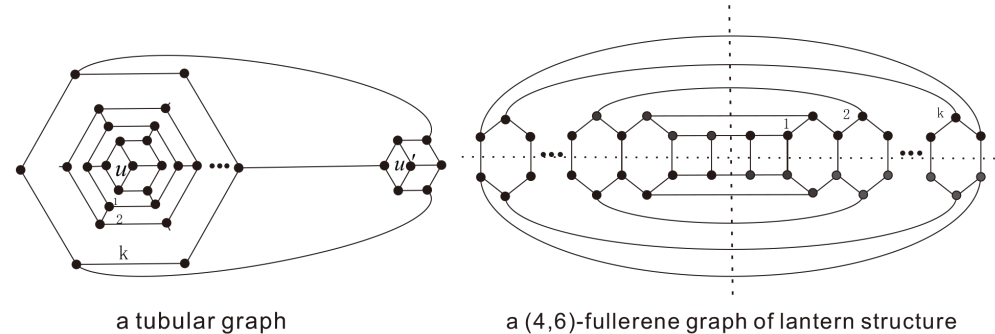

Figure 1. The $(4,6)$-fullerenes in Lemma 1.

Proof. Let $G$ be a cube. Checking the structure of $G$, we obtain that the eccentricity of every vertex in $G$ is 3. By (1) and (2), we have

$$
\xi^{c e}(G)=\sum_{v \in V(G)} \frac{d(v)}{\varepsilon(v)}=\sum_{v \in V(G)} \frac{3}{3}=8
$$

and

$$
\xi^{c}(G)=\sum_{v \in V(G)} d(v) \varepsilon(v)=\sum_{v \in V(G)} 3 \times 3=72 .
$$

Similarly, let $G$ be a hexagonal prism. Checking the structure of $G$, we obtain that the eccentricity of every vertex in $G$ is 4 . By (1) and (2), we obtain

$$
\xi^{c e}(G)=\sum_{v \in V(G)} \frac{d(v)}{\varepsilon(v)}=\sum_{v \in V(G)} \frac{3}{4}=9,
$$

and

$$
\xi^{c}(G)=\sum_{v \in V(G)} d(v) \varepsilon(v)=\sum_{v \in V(G)} 3 \times 4=144
$$

Theorem 3 Let $G$ be a tubular graph, and let the number of hexagon-layer in G be $k \geq 1$. Then

$$
\begin{aligned}
\xi^{c e}(G) & =18 \sum_{i=3}^{k+4} \frac{1}{k+i}+\frac{6}{2 k+5} ; \\
\xi^{c}(G) & =27 k^{2}+129 k+156 .
\end{aligned}
$$

Proof. Let $v$ be a vertex of $G$. By the structure of $G$ (see Figure 1), we know that the eccentricity of $v$ equal to the distance between $v$ and $u$ (or $u^{\prime}$ ). Thus, the eccentricity sequence of $G$ is $(\overbrace{2 k+5,2 k+5}^{2}, \overbrace{2 k+4, \cdots, 2 k+4}^{6}, \overbrace{2 k+3, \cdots, 2 k+3}^{6}$, $\cdots, \overbrace{k+4, \cdots, k+4}^{6}, \overbrace{k+3, \cdots, k+3}^{6}$ ). By (1) and (2), we obtain

$$
\xi^{c e}(G)=\sum_{v \in V(G)} \frac{d(v)}{\varepsilon(v)}
$$




$$
\begin{gathered}
=3\left[\left(\frac{1}{2 k+4}+\frac{1}{2 k+3}+\cdots+\frac{1}{k+3}\right) \times 6+\frac{1}{2 k+5} \times 2\right] \\
=18 \sum_{i=3}^{k+4} \frac{1}{k+i}+\frac{6}{2 k+5} .
\end{gathered}
$$

and

$$
\begin{gathered}
\xi^{c}(G)=\sum_{v \in V(G)} d(v) \varepsilon(v) \\
=3[2(2 k+5)+6[(k+3)+(k+4)+\cdots+(2 k+4)]] \\
=3\left[2(2 k+5)+6 \frac{[(k+3)+(2 k+4)](k+2)}{2}\right] \\
=27 k^{2}+129 k+156 .
\end{gathered}
$$

This completes the proof.

Theorem 4 Let $G$ be a $(4,6)$-fullerene graph having lantern structure, and let the number of hexagon-layer in $G$ be $k \geq 1$, see Figure 1 . Then

$$
\begin{gathered}
\xi^{c e}(G)=24 \sum_{i=3}^{k+3} \frac{1}{k+i} ; \\
\xi^{c}(G)=36 k^{2}+108 k+72 .
\end{gathered}
$$

Proof. Checking $G$, we see that $G$ is symmetry. By the symmetry of $G$, it is easy to obtain that the eccentricity sequence of $G$ is $(\overbrace{2 k+3, \cdots, 2 k+3}^{8}$, $\overbrace{2 k+2, \cdots, 2 k+2}^{8}, \cdots, \overbrace{k+4, \cdots, k+4}^{8}, \overbrace{k+3, \cdots, k+3}^{8}$ ). By (1) and (2), we have

$$
\begin{gathered}
\xi^{c e}(G)=\sum_{v \in V(G)} \frac{d(v)}{\varepsilon(v)} \\
=3\left[\left(\frac{1}{2 k+3}+\frac{1}{2 k+2}+\cdots+\frac{1}{k+3}\right) \times 8\right] \\
=24 \sum_{i=3}^{k+3} \frac{1}{k+i} .
\end{gathered}
$$

and

$$
\begin{gathered}
\xi^{c}(G)=\sum_{v \in V(G)} d(v) \varepsilon(v) \\
=3[8[(k+3)+(k+4)+\cdots+(2 k+2)+(2 k+3)]] \\
=3\left[8 \frac{[(k+3)+(2 k+3)](k+1)}{2}\right] \\
=36 k^{2}+108 k+72 .
\end{gathered}
$$

The proof of the theorem is now complete.

\section{Conclusion}

In this note, we give calculation formulas of ECI and CEI of all $(4,6)$-fullerenes 
with regular structures, respectively. In the future, we will discuss the calculation of ECI and CEI of a $(4,6)$-fullerene graph of dispersive structure, and try to give an algorithm.

\section{Conflict of Interests}

The authors declare that there is no conflict of interests regarding the publication of this paper.

\section{Acknowledgements}

The work is supported by the Natural Science Foundation of Qinghai Province (2016-ZJ-947Q), the Ministry of Education Chunhui Project (No. Z2017047) and the High-level Personnel of Scientific Research Project of QHMU (2016XJG07).

\section{References}

[1] Sharma, V., Goswami, R. and Madan, A.K. (1997) Eccentric Connectivity index: A Novel Highly Discriminating Topological Descriptor for Structure-Property and Structure-Activity Studies. Journal of Chemical Information and Computer Sciences, 37, 273-282. https://doi.org/10.1021/ci960049h

[2] Ashrafi, A.R., Došlić, T. and Saheli, M. (2011) The Eccentric Connectivity Index of TUC4C8(R) Nanotubes. MATCH Communications in Mathematical and in Computer Chemistry, 65, 221-230.

[3] Došlić, T., Saheli, M. and Vukicevic, D. (2010) Eccentric Connectivity Index: Extremal Graphs and Values. Iranian Journal of Mathematical Chemistry, 1, 45-56.

[4] Dureja, H., Gupta, S. and Madan, A.K. (2008) Predicting Anti-HIV-1 Activity of 6-Arylbenzonitriles: Computational Approach Using Superaugmented Eccentric Connectivity Topochemical Indices. Journal of Molecular Graphics, 26, 1020-1029. https://doi.org/10.1016/j.jmgm.2007.08.008

[5] Yu, G., Feng, L. and Ilić, A. (2011) On the Eccentric Distance Sum of Trees and unicyclic Graphs. Journal of Mathematical Analysis and Applications, 375, 99-107. https://doi.org/10.1016/j.jmaa.2010.08.054

[6] Zhang, J., Zhou, B. and Liu, Z. (2012) On the Minimal Eccentric Connectivity Indices of Graphs. Discrete Mathematics, 312, 819-829. https://doi.org/10.1016/j.disc.2011.10.006

[7] Zhou, B. and Du, Z. (2010) On Eccentric Connectivity Index. MATCH Communications in Mathematical and in Computer Chemistry, 63, 181-198.

[8] Gupta, S., Singh, M. and Madan, A.K. (2000) Connective Eccentricity Index: A Novel Topological Descriptor for Predicting Biological Activity. Journal of Molecular Graphics and Modelling, 18, 18-25. https://doi.org/10.1016/S1093-3263(00)00027-9

[9] Azari, M. and Iranmanesh, A. (2013) Computing the Eccentric-Distance Sum for Graph Operations. Discrete Applied Mathematics, 161, 2827-2840. https://doi.org/10.1016/j.dam.2013.06.003

[10] Li, S., Zhang, M., Yu, G. and Feng, L. (2012) On the Extremal Values of the eccentric Distance Sum of Trees. Journal of Mathematical Analysis and Applications, 390, 99-112. https://doi.org/10.1016/j.jmaa.2012.01.022

[11] Yu, G. and Feng, L. (2013) On the Connective Eccentricity Index of Graphs. MATCH 
Communications in Mathematical and in Computer Chemistry, 69, 611-628.

[12] Yu, G., Qu, H., Tang, L. and Feng, L. (2014) On the Connective Eccentricity Index of Trees and Unicyclic Graphs with Given Diameter. Journal of Mathematical Analysis and Applications, 420, 1776-1786. https://doi.org/10.1016/j.jmaa.2014.06.050

[13] Xu, K., Das, K. and Liu, H. (2016) Some Extremal Results on the Connective Eccentricity Index of Graphs. Journal of Mathematical Analysis and Applications, 433, 803-817. https://doi.org/10.1016/j.jmaa.2015.08.027

[14] Xu, K., Liu, M., Das, K., Gutman, I. and Furtula, B. (2014) A Survey on Graphs Extremal with Respect to Distance-Based Topological Indices. MATCH Communications in Mathematical and in Computer Chemistry, 71, 461-508.

[15] Gao, Y. and Zhang, H. (2014) Clar Structure and Fries Set of Fullerenes and (4,6)-Fullerenes. Journal of Applied Mathematics, 2014, Article ID: 196792. https://doi.org/10.1155/2014/196792

[16] Jiang, X. and Zhang, H. (2011) On Forcing Matching Number of Boron-Nitrogen Fullerene Graphs. Discrete Applied Mathematics, 159, 1581-1593. https://doi.org/10.1016/j.dam.2011.05.006

[17] Zhang, H. and Liu, S. (2010) 2-Resonance of Plane Bipartite Graphs and Its Applications to Boron-Nitrogen Fullerenes. Discrete Applied Mathematics, 158, 1559-1569. https://doi.org/10.1016/j.dam.2010.05.012

[18] Wei, Z. and Zhang, H. (2017) 2-Resonance of Plane Bipartite Graphs and Its Applications to Boron-Nitrogen Fullerenes. MATCH Communications in Mathematical and in Computer Chemistry, 77, 707-724. 